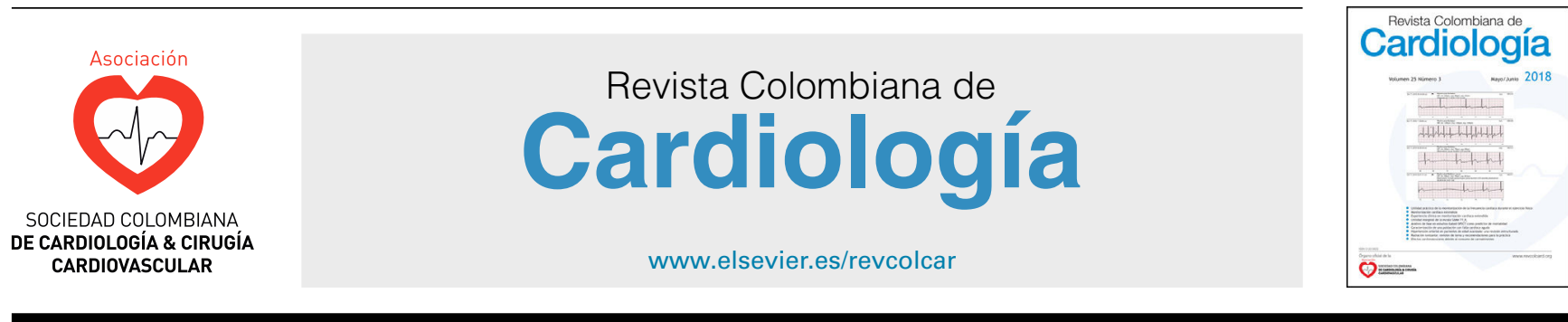

HISTORIA DE LA CARDIOLOGÍA

\title{
Mi encuentro con la electrofisiología cardiaca
}

\section{My memories in cardiac electrophysiology}

\author{
Víctor Manuel Velasco-Caicedo
}

\author{
Departamento de Electrofisiología y Estimulación Cardiaca. Fundación Clínica Shaio, Bogotá, Colombia
}

Recibido el 17 de julio de 2020; aceptado el 17 de julio de 2020

Disponible en Internet el 15 de septiembre de 2020

Tuve la fortuna de vivir cinco años maravillosos e inolvidables en México (1976-1980). En Ciudad de México realicé mis entrenamientos en cardiología en el Instituto Nacional de Cardiología (INC), y de Medicina Crítica y Cuidado Intensivo en el American British Cowdray Hospital. En ese país me apasioné por la electrocardiografía y la electrofisiología; la escuela mexicana de electrocardiografía deductiva era reconocida mundialmente $e^{1,2}$. No conocí a su principal mentor, Demetrio Sodi Pallares, que ya se había retirado del INC, pero los otros grandes de la electrocardiografía mexicana, Gustavo Medrano, Abdo Bisteni y Alfredo de Micheli, fueron mis profesores.

Los primeros trabajos que realicé en ese mundo estuvieron relacionados con los hallazgos electrocardiográficos en las enfermedades pulmonares crónicas, particularmente en la tromboembolia pulmonar a repetición, que fue mi tesis de grado con el doctor Víctor Manuel Alatriste ${ }^{3}$. Otros trabajos electrocardiográficos fueron los que publiqué con el doctor Mario Shapiro ${ }^{4}$, sobre la utilidad de las derivaciones precordiales V3R y V4R en el infarto agudo del ventrículo derecho.

En electrofisiología cardíaca los aportes de la escuela mexicana han sido significativos. Por ejemplo, en 1958, Jesús Alanís y colaboradores describieron la actividad eléctrica del haz de His en el corazón aislado y perfundido del perro y lo llamaron potencial $\mathrm{H}^{5}$. Por esa época se formó

\footnotetext{
Correo electrónico: velascovm@gmail.com
}

como cardiólogo en el INC el doctor Paul Puech, quien luego regresó a su país, Francia, y en la Clínica St. Eloi de Montpellier, en 1960, en compañía de los doctores Giraud y Latour, demostraron por primera vez la actividad eléctrica del haz de His en el hombre ${ }^{6,7}$.

Uno de mis compañeros mexicanos de Residencia en el INC, el doctor Jorge Vidal-Gárate, se había entrenado en electrofisiología cardíaca con el doctor Paul Puech en Francia. Hizo empatía con el doctor Manuel Cárdenas, jefe de la Unidad de Cuidado Coronario, autor de un Manual de Arritmias $^{8}$, y logró consolidar su idea de desarrollar, en un salón anexo a la Unidad Coronaria, el primer laboratorio invasivo de electrofisiología cardíaca en humanos del INC con el equipamiento básico: un intensificador de imagen portátil, un polígrafo y un aparato para estimulación cardíaca programada. Los estudios eran ocasionales. En esa época el laboratorio para electrocardiografía experimental en perros del INC tenía mejores salones y equipos. Era más importante la electrocardiografía que la electrofisiología.

Después ingresó como profesor al INC, en 1980, el doctor Luis Molina, quien también se había formado como electrofisiólogo con el doctor Paul Puech en Montpellier (1976-1979). Su lucha fue con el servicio de hemodinamia, pero logró consolidar una sala o laboratorio exclusivo para electrofisiología cardíaca e iniciar un programa formal de entrenamiento en la especialidad.

A finales de 1980, regresé a Colombia y reflexionando ahora (2020), cuarenta años después, lo que hice en mi país fue repetir la historia de México. 
Empecé a trabajar en la entonces Clínica Santa Rosa de la Caja Nacional de Previsión Social (Cajanal) de Bogotá, hoy Hospital Universitario Nacional de Colombia, como cardiólogo-intensivista, y logré transformar un salón adjunto a la unidad de cuidado intensivo ( $\mathrm{UCI})$, donde dormían los médicos intensivistas de turno, en una sala o laboratorio de electrofisiología cardiaca invasiva con intensificador de imagen portátil, polígrafo y estimulador cardíaco programado; similar a la de los doctores Vidal y Cárdenas en México. Todo esto fue posible gracias al entusiasta e incondicional apoyo en Cajanal de los doctores Eduardo García Vargas, Jefe de la UCI, y Enrique Alvarado Acevedo, Jefe de Cardiología.

Un año después, se inauguró en Cajanal la primera sala de hemodinamia de la institución, la cual fue dotada con equipos muy modernos para la época, y allí se me permitió realizar estudios electrofisiológicos. La historia del laboratorio de electrofisiología de Cajanal la divido en dos etapas, la primera, entre 1981 y 1982, que se caracterizó por arreglos en la sala, compra de equipos (polígrafo, estimulador, catéteres, etc.), algunos estudios electrofisiológicos y formación de personal paramédico y médico. Realicé viajes de entrenamiento a Ciudad de México con el doctor Luis Molina, a Montpellier con el doctor Paul Puech y al Hospital General del Sur en Maracaibo, Venezuela, con el doctor Bolívar Portillo, donde trabajé como profesor visitante. La segunda etapa, a partir de agosto de 1982, ya con el equipo necesario completo y planta física adecuada, fue la etapa de funcionamiento regular, continuo, con un promedio de dos estudios electrofisiológicos semanales. En hemodinamia y cardiología en Cajanal fueron admirables colaboradores los doctores Alcides Houghton y Alberto Quintero. Esa es la historia del primer laboratorio de electrofisiología cardíaca que funcionó regularmente en Colombia?.

Los primeros trabajos científicos realizados en Cajanal fueron sobre búsqueda de enfermedad del nodo sinusal en pacientes con bradicardia sinusal y bradiarritmias, en 30 pacientes, con pruebas de función sinusal y de conducción aurículo-ventricular invasivas similares a las que se practican hoy en día, que presenté en el VII Congreso Colombiano de Medicina Interna, en Medellín, en octubre de $1982^{10}$ y el trabajo sobre Estudios electrofisiológicos en taquicardia ventricular sostenida, con 9 pacientes, que se presentó en el VIII Congreso Colombiano de Medicina Interna, en Barranquilla, en octubre de $1984^{11}$. En un congreso colombiano de cardiología premiaron nuestro trabajo sobre efectos electrofisiológicos del sulfato de magnesio en humanos ${ }^{12}$.

Cuando visité el servicio de electrofisiología de la Universidad de Alabama, en Birmingham, Estados Unidos, que dirigía el doctor Albert Waldo, en 1983, conocí al doctor Álvaro Mesa Arroyave, médico colombiano, formado en Cardiología en el Instituto Nacional de Cardiología de México, quien, por esa época, realizaba un fellow en electrofisiología. El doctor Mesa regresó a Medellín y en el Centro Cardiovascular Colombiano "Clínica Santa María", hoy Clínica CardioVid, inició, en 1985, el segundo laboratorio de electrofisiología que existió y existe en Colombia ${ }^{13}$.

A finales de 1981, en forma simultánea o paralela con mi oficio en Cajanal, comencé a trabajar como jefe de la unidad de cuidado intensivo (UCI) de la Fundación Clínica Shaio, en Bogotá, con la condición de permitir mi trabajo en electrofisiología en Cajanal mientras se implementaba esta especialidad en Shaio. El proceso fue lento. En la $\mathrm{UCl}$, mis alumnos y luego compañeros de trabajo, Marco Perafán y Daniel Isaza, respaldaron mis escapadas a Cajanal. En la Shaio, en el servicio de hemodinamia, me facilitaron la sala más vieja para mis experimentos electrofisiológicos y en el servicio de radiología en una mesa que se podía inclinar a 60 - 70 grados realicé las primeras pruebas de mesa inclinada o basculante que se practicaron en Colombia ${ }^{14}$. La experiencia inicial de fulguración o ablación de His con corriente directa la hicimos con casos de Cajanal y Shaio (1989-1991). Después se desarrolló la ablación con energía de radiofrecuencia.

Todo cambió en 1991 cuando la Fundación Clínica Shaio, entidad pionera y especializada en enfermedades cardiovasculares, adquirió un moderno polígrafo Mingograf 7 de Siemens, un estimulador Medtronic modelo 5326 y los catéteres necesarios para realizar estudios electrofisiológicos. En marzo de ese año, empecé a realizar regularmente, casi todos los días, estudios electrofisiológicos en la Shaio. La primera enfermera jefe de tiempo completo que me acompañó en electrofisiología fue Luisa Laverde. En 1994 ingresó al servicio el doctor Fernando Rosas después de terminar su entrenamiento de postgrado en electrofisiología en el Hospital Jean Rostand, de París, Francia, con los doctores Guy Fontaine y Robert Frank. Desde entonces trabajamos en equipo.

El desarrollo de la electrofisiología y la estimulación cardiaca en Shaio, en Colombia y en el mundo ha sido formidable en los últimos años y será motivo de otro artículo. En este trabajo solo quise consignar mi experiencia inicial.

\section{Conflicto de intereses}

Ninguno.

\section{Bibliografía}

1. Sodi Pallares D, Bisteni A, Medrano GA. Electrocardiografía y vectocardiografía. México: La Prensa Médica Mexicana;. 1964.

2. Sodi Pallares D, Medrano G, Bisteni A, Ponce de León J. Electrocardiografía clínica. Análisis deductivo. México: Ediciones del Instituto Nacional de Cardiología de México;. 1968.

3. Alatriste VM, Velasco VM, Medrano G, Villareal A. Tromboembolia pulmonar de repetición Correlación entre los hallazgos electrocardiográficos y hemodinámicos. Arch Inst Cardiol Mex. 1981;51:453-62.

4. Velasco VM, Shapiro M, Martínez J. Búsqueda electrocardiográfica de los infartos agudos del ventrículo derecho. Arch Inst Cardiol Mex. 1981;15:75-82.

5. Alanis J, González H, López E. The electrical activity of the bundle of His. J Physiol. 1958;142:127.

6. Giraud G, Latour H, Puech P. Láctivité du noued de Tawara e du faisceau de His en électrocardiographie chez lhomme. Mal Cardiovasc. 1960;1:321.

7. Giraud G, Puech P, Latour H, Hertault J. Variations de potentiel liées a láctivité du systeme de conduction auriculo-ventriculaire chez lhomme. Enreistrement électrocardiographique endocavitaire. Arch Mal Coeur. 1960;53:757.

8. Cardenas LM. Clínica de las arritmias, 2aㅡ. ed. México: La Prensa Médica Mexicana;. 1987.

9. Velasco VM. Historia del laboratorio de electrofisiología cardíaca de la Caja Nacional de Previsión Social de Bogotá Colombia. En: Reynolds J, editor. 30 años de la estimulación cardíaca en Colombia. Bogotá: Editorial Andes; 1988. p. 85-6. 
10. Velasco VM. Búsqueda de enfermedad del nodo sinusal en pacientes con bradicardia sinusal. Acta Med Colomb. 1982.

11. Velasco VM. Estudios electrofisiológicos en taquicardia ventricular. Acta Med Colomb. 1984.

12. Velasco VM. Efectos electrofisiológicos del sulfato de magnesio en humanos. Rev Col Cardiol. 1990;3:88.
13. Mesa A. Electrofisiología. En: Reynolds J, editor. 30 años de la estimulación cardíaca en Colombia. Bogotá: Editorial Andes; 1988. p. 95-8.

14. Velasco VM. Utilidad de la mesa basculante en pacientes con síncope de origen inexplicable. Rev Col Cardiol. 1993;4:93-7. 